\title{
TURBULENCE AND STOCHASTIC STAR FORMATION IN THE LARGE MAGELLANIC CLOUD
}

\author{
J.V. FEITZINGER, J. SPICKER \\ Astronomical Institut, Ruhr-University \\ Bochum and Observatory of the City of Bochum \\ Castroper Str. 67 \\ D-4630 Bochum \\ F R Germany
}

\begin{abstract}
A recent turbulence analysis of the Large Magellanic Cloud (LMC) is summarized. New model calculations on stochastic self-propagating star formation taking into account the multi-phase structure of the interstellar medium are presented. The relationship between star formation and turbulence is discussed.
\end{abstract}

From an analysis of the two-dimensional auto-correlation and structure function of HI, HII, radiocontinuum, IR (IRAS) emission and colour excess, the different scale properties of the emission regions and of turbulence in the LMC are derived. The microscales of turbulence range from $300 \mathrm{pc}$ to $400 \mathrm{pc}$, with integral scale sizes being about 500 pc (Figs. $1 \&$ 2). Star-forming activity and the velocity structure of supergiant shells are correlated with these scales (Spicker \& Feitzinger 1988a, b).

From the appearance of the supershells in the auto-correlation maps (Fig. 2) it is concluded that they are most likely to influence the interstellar medium dynamically. The supershells produce the observed structures on the largest scale sizes and trigger star formation. The structuring of the interstellar medium by self-propagating star-formation processes fits well into this picture. The multiphase simulations of the interstellar medium realistically produce the morphology of the LMC (Feitzinger 1987).

\section{References}

Feitzinger, J.V., Haynes, R.F., Klein, U., Wielebinski, R., Perschke, M. (1987), Vistas in Astronomy, 30, 243.

Spicker, J., Feitzinger, J.V. (1988a), Astron. Astrophys. 191, 10.

Spicker, J., Feitzinger, J.V. (1988b), Astron. Astrophys. 191, 186. 


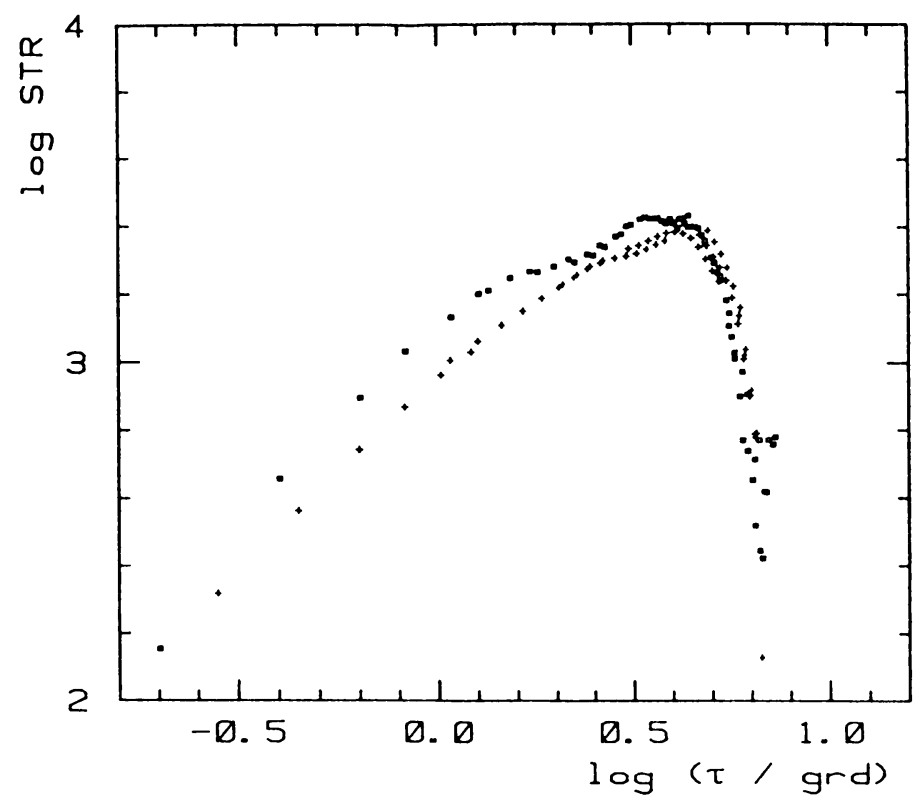

Figure 1. Radial cuts through the two-dimensional rotation-free radial velocity field. One cut (+) runs in the direction of the supershell LMC4; the other (d) in the direction of LMC2 and LMC3. The slope is $\sim 1.3$.

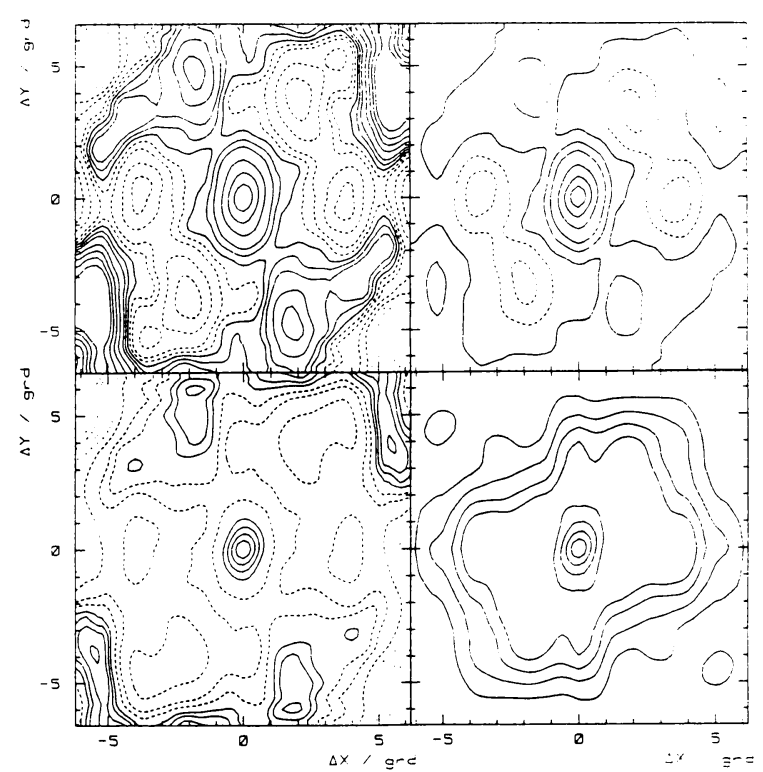

Figure 2. Two-dimensional autocorrelation and structure function of the rotation-free radial velocity field. Anisotropic recorrelations can be found in the direction of the bar (upper) and the supershells (lower panel). 